\title{
Homogenization of the G-equation: a metric approach
}

\section{Antonio Siconolfi ${ }^{1}$}

Accepted: 27 April 2021 / Published online: 30 June 2021

(c) The Author(s) 2021

\begin{abstract}
The aim of the paper is to recover some results of Cardaliaguet-Nolen-Souganidis in Cardaliaguet et al. (Arch Rat Mech Anal 199(2): 527-561, 2011) and Xin-Yu in Xin and Yu (Commun Math Sci 8(4): 1067-1078, 2010) about the homogenization of the G-equation, using different and simpler techniques. The main mathematical issue is the lack of coercivity of the Hamiltonians. In our approach we consider a multivalued dynamics without periodic invariants sets, a family of intrinsic distances and perform an approximation by a sequence of coercive Hamiltonians.
\end{abstract}

Keywords Homogenization · Hamilton-Jacobi equations · Effective Hamiltonian

Mathematics Subject Classification 35B40 $\cdot 35 \mathrm{~F} 21 \cdot 37 \mathrm{~J} 99 \cdot$ 49L25

\section{Introduction}

True to the title, the aim of these notes is to study the homogenization of time dependent Hamilton-Jacobi equations with Hamiltonian

$$
H(x, p)=|p|+p \cdot V(x) \quad(x, p) \in \mathbb{R}^{N} \times \mathbb{R}^{N},
$$

where the advection field $V$ is $\mathbb{Z}^{N}$-periodic, Lipschitz continuous and with suitably small divergence. This Hamiltonian appears in combustion theory, where the homogenization result can be interestingly interpreted, see [3,9]. However, we do not treat this side of the issue, and stay solely concerned with the theoretical aspects of the matter.

As it is well known, the relevant mathematical point is that $H$ looses coercivity whenever $|V| \geq 1$ and consequently, as pointed out in [3,9], there could be no homogenization if $V$ is just assumed to be Lipschitz continuous or even more regular, some additional requirements are

\footnotetext{
"This article is part of the topical collection "Viscosity solutions-Dedicated to Hitoshi Ishii on the award of the 1st Kodaira Kunihiko Prize" edited by Kazuhiro Ishige, Shigeaki Koike, Tohru Ozawa, and Senjo Shimizu.".

Antonio Siconolfi

siconolf@mat.uniroma1.it

1 Department of Mathematics, Università degli Studi di Roma "La Sapienza", Piazzale Aldo Moro 5, 00185 Rome, Italy
} 
needed, specifically the condition on the divergence. This is in contrast to the homogenization theory for coercive Hamiltonians, see [7].

It is opportune to make clear that our outputs are not new, in [3] it has been already established, among other things, that homogenization takes place under assumptions just slightly different from ours, and for vector fields $V$ also depending on time, which adds further complications.

The interest of our contribution, if any, is that the proofs are different and, we believe, more simple and intuitive. They rely on a metric approach to the matter. Note that metric techniques have been already used in homogenization of Hamilton-Jacobi equations, in particular in the stationary ergodic case, see for instance [2,5].

To begin with, we define the effective Hamiltonian $\bar{H}$, appearing in the limit equation, as

$$
\bar{H}(P)=\inf \{a \mid H(x, D u+P)=a \text { admits an usc bdd periodic subsolution }\},
$$

without passing through ergodic approximation. From this formulation, we see that the subsolutions of $H(x, D u+P)=\bar{H}(P)+\delta$, for $\delta>0$ small, can be used as approximate correctors to prove, via the perturbed test function method, see [4], that the weak lower semilimit of the solutions to the $\varepsilon$-oscillatory equations is supersolution of the limit equation with the effective Hamiltonian. This depends on the very definition of $\bar{H}$, and can be done without using the assumption on the divergence of $V$.

The solution of the problem therefore boils down to show the existence of lsc bounded periodic supersolutions to the critical equation $H(x, D u+P)=\bar{H}(P)$. This should actually allow performing the other half of the homogenization procedure.

Backtracking the issue, we see that such supersolutions do exist provided that some periodic distances related to $H$ are bounded. In other terms we discover, not surprisingly, that some controllability condition is needed in order for homogenization to take place, compare for instance with [1], where other noncoercive models are considered.

The boundedness of the distances is in turn equivalent, see Sects. 5-6, to the nonexistence of periodic invariant sets for a multivalued dynamics related to $H$. This is the key point where the condition on the divergence of $V$ enters into play.

The key point in this respect is Theorem 4.2, where it is proved that if such a set, say $\Omega$, does exist, then it possesses Lipschitz boundary, but then the compression of $V$ toward the interior of $\Omega$ is not compatible with its small divergence. This result is deduced through divergence theorem and isoperimetric inequality, see Lemma 4.12, Theorem 4.9. More precisely, we apply the isoperimetric inequality on the flat torus $\mathbb{T}^{N}=\mathbb{R}^{N} / \mathbb{Z}^{N}$, so that our condition on $V$ reads

$$
\|\operatorname{div} V\|_{L^{N}\left(\mathbb{T}^{N}\right)} \leq \frac{1}{\chi}
$$

where $\chi$ is the isoperimetric constant on $\mathbb{T}^{N}$, which coincides with that of $Q_{\frac{1}{2}}=(0,1 / 2)^{N}$, see Sect. 2. This is actually the same assumption of [3] except that they use the isoperimetric constant of $Q_{1}=(0,1)^{N}$. We are not aware of comparison results between these two constants, and we do not know if this difference is just a technical detail, or some more deep fact is involved.

The paper is organized as follows: in Sect. 2 we give notations and preliminary results. In Sect. 3 we write down the problem with the standing assumptions. Section 4 is devoted to the definition of a relevant multivalued dynamics and the proof of the nonexistence of periodic invariant sets. In Sect. 5 we define some distances intrinsically related to $H$ and study their properties. In Sect. 5 we approximate $H$ by a sequence $H_{k}$ of coercive Hamiltonians and prove that the effective Hamiltonians $\bar{H}_{k}$ converge to $\bar{H}$. From this result we deduce the 
existence of lsc periodic bounded supersolutions to the critical equation related to $H$, and complete the proof of the homogenization result. Finally, in the Appendix we present some proofs.

\section{Preliminaries}

Given a subset $E$ of $\mathbb{R}^{N}$, we write $\bar{E}$, int $E, \partial E, E^{c}$ to indicate its closure, interior, boundary, complement, respectively. For $x \in \mathbb{R}^{N}, r>0$, we denote by $\mathcal{B}(x, r)$ the open ball centered at $x$ with radius $r$. Given $a>0$, we denote by $Q_{a}$ the open hypercube $(0, a)^{N}$. We write $\mathbb{T}^{N}=\mathbb{R}^{N} / \mathbb{Z}^{N}$ to indicate the flat torus. Namely the torus with the metric induced by the Euclidean one on $\mathbb{R}^{N}$. With this choice $\mathbb{R}^{N}$ and $\mathbb{T}^{N}$ are locally isometric.

We identify $\mathbb{R}^{N}$ and its dual, and use the symbol · to denote both the duality pairing and the scalar product.

Given a convex subset $C$ of $\mathbb{R}^{N}$, the negative polar cone $C^{-}$is defined through

$$
C^{-}=\left\{p \in \mathbb{R}^{N} \mid p \cdot q \leq 0 \text { for any } q \in C\right\} .
$$

All the curves we will consider are assumed to be Lipschitz continuous.

The acronyms usc /lsc stand for upper/lower semicontinuous. For any bounded function $u: \mathbb{R}^{N} \rightarrow \mathbb{R}$, and $\delta>0$ we define $\delta$-inf and sup convolutions, respectively, as follows:

$$
\begin{aligned}
& u_{\delta}(x)=\inf \left\{u(y)+\frac{1}{2 \delta}|x-y|^{2} \mid y \in \mathbb{R}^{N}\right\} \\
& u^{\delta}(x)=\sup \left\{u(y)-\frac{1}{2 \delta}|x-y|^{2} \mid y \in \mathbb{R}^{N}\right\}
\end{aligned}
$$

Given $x_{0} \in \mathbb{R}^{N}$, we say that $y_{0}$ is $u_{\delta}$-optimal with respect to $x_{0}$ if $y_{0}$ realizes the infimum in the formula of inf convolution. The notion of $u^{\delta}$-optimal point is given similarly.

Given a sequence of locally bounded functions $u_{n}$, we define the lower/upper weak semilimit as follows

$$
\begin{aligned}
\liminf \operatorname{in}_{n}(x) & =\inf \left\{\lim \inf \left\{u_{n}\left(x_{n}\right) \mid x_{n} \rightarrow x\right\}\right. \\
\lim \sup ^{\#} u_{n}(x) & =\sup \left\{\lim \sup \left\{u_{n}\left(x_{n}\right) \mid x_{n} \rightarrow x\right\}\right.
\end{aligned}
$$

The term super/sub solution for a given PDE must be understood in the viscosity sense. We denote by $D^{+} u(x)$ (resp. $D^{-} u(x)$ ) the viscosity superdifferential (resp. subdifferential).

Definition 2.1 We say that a subset $\Omega \subset \mathbb{R}^{N}$ has Lipschitz boundary if for any $x \in \partial \Omega$ there is a neighborhood $U$, an hyperplane of the form $x+p^{\perp}$, for some nonvanishing vector $p$, and a Lipschitz function $\psi$ defined in $\left(x+p^{\perp}\right) \cap U$ with

(i) $\partial \Omega \cap U=\left\{y+\psi(y) p \mid y \in\left(x+p^{\perp}\right) \cap U\right\}$

(ii) int $\Omega \cap U=\left\{y+\lambda p \mid y \in\left(x+p^{\perp}\right) \cap U, \lambda>\psi(y)\right\}$

The isoperimetric constant $\chi$ on the torus $\mathbb{T}^{N}$ is the smallest constant such that

$$
|\Theta|^{1-1 / N} \leq \chi \mathcal{H}^{N-1}(\partial \Theta)
$$

for any subset $\Theta$ of $\mathbb{T}^{N}$ with Lipschitz boundary. Here $\mathcal{H}^{N-1}$ stands for the $(N-1)$ dimensional Hausdorff measure and $|\cdot|$ for the $N$-dimensional Lebesgue measure. 
The (relative) isoperimetric constant $\chi_{a}$ on $Q_{a}$ is the smallest constant such that

$$
|\Theta|^{1-1 / N} \leq \chi_{a} \mathcal{H}^{N-1}\left(\partial \Theta \cap Q_{a}\right)
$$

for all subset $\Theta \subset Q_{a}$ with Lipschitz boundary.

According to [8], the isoperimetric problems in $\mathbb{T}^{N}$ in $Q_{\frac{1}{2}}$ are equivalent, and consequently the constants $\chi$ and $\chi_{\frac{1}{2}}$ coincide.

A formulation of the divergence Theorem on the torus is:

Let $\Theta \subset \mathbb{T}^{N}$ be a set with Lipschitz boundary and $V: \mathbb{T}^{N} \rightarrow \mathbb{R}^{N}$ a Lipschitz continuous vector field, then

$$
\int_{\Theta} \operatorname{div} V d x=\int_{\partial \Theta} V \cdot n_{\Theta} d \mathcal{H}^{N-1},
$$

where $n_{\Theta}$ is the outward unit normal on $\partial \Theta$.

Remark 2.2 We recall that if $\Theta$ has Lipschitz boundary then the outer normal $n_{\Theta}(x)$ exists for $\mathcal{H}^{N-1}$ a.e. $x \in \partial \Theta$ and satisfies the following property:

$$
\operatorname{proj}_{\Theta}\left(x+t n_{\Theta}(x)\right)=x \quad \text { for } t>0 \quad \text { suitably small, }
$$

where $\operatorname{proj}_{\Theta}$ indicates the projection on $\bar{\Theta}$.

\section{The problem}

We consider the Hamiltonian

$$
H(x, p)=|p|+p \cdot V(x) \quad \text { in } \mathbb{R}^{N} \times \mathbb{R}^{N},
$$

where $V: \mathbb{R}^{N} \rightarrow \mathbb{R}^{N}$, is a vector field that we assume

(A1) $\mathbb{Z}^{N}$-periodic and Lipschitz continuous;

(A2) satisfying $\|\operatorname{div} V\|_{L^{N}\left(\mathbb{T}^{N}\right)} \leq \frac{1}{\chi}$, where $\chi$ is the isoperimetric constant in $Q_{\frac{1}{2}}$ and in $\mathbb{T}^{N}$ as well, see Sect. 2 .

Throughout the paper we will denote by $L_{V}, M_{V}$, the Lipschitz constant of $V$ and the maximum of $|V(x)|$, respectively. We consider, for $\varepsilon>0$, the family of time-dependent Hamilton-Jacobi equations

$$
\left\{\begin{array}{cl}
\left(u_{\varepsilon}\right)_{t}(x / \varepsilon, t)+H\left(x / \varepsilon, D u_{\varepsilon}\right)=0 & \text { in } \mathbb{R}^{N} \times(0,+\infty) \\
u_{\varepsilon}(\cdot, 0) & =u_{0} \quad \text { in } \mathbb{R}^{N}
\end{array}\right.
$$

where

(A3) $u_{0}$ is bounded uniformly continuous.

Our goal is to study the asymptotic behavior of the (unique) solutions $u^{\varepsilon}$ of $\left(\mathrm{HJ}_{\varepsilon}\right)$, as $\varepsilon$ goes to 0 , and to prove that they locally uniformly converges to a function $u^{0}$ which is (unique) solution of a problem of the form

$$
\left\{\begin{array}{cl}
u_{t}(x, t)+\bar{H}(D u)=0 & \text { in } \mathbb{R}^{N} \times(0,+\infty) \\
u(\cdot, 0) & =u_{0} \quad \text { in } \mathbb{R}^{N}
\end{array}\right.
$$

where $\bar{H}$ is a suitable limit Hamiltonian convex and positively homogeneous.

Conditions $\left(A_{1}\right),\left(A_{2}\right),\left(A_{3}\right)$ will be assumed throughout the paper, without any further mentioning. 


\section{Invariant sets}

We introduce a set-valued vector field related to $V$. We set for any $x \in \mathbb{R}^{N}$,

$$
F(x)=F_{1}(x)=\overline{\operatorname{co}}\{\mathcal{B}(V(x), 1) \cup\{0\}\},
$$

where $\overline{\mathrm{co}}$ stands for closed convex hull. We also define for $\delta \in(0,1)$

$$
F_{\delta}(x)=\overline{\operatorname{co}}\{\mathcal{B}(V(x), \delta) \cup\{0\}\} .
$$

The set-valued vector fields $F_{\delta}(x)$ are Hausdorff-continuous with compact convex values for any $\delta \in(0,1]$. We will say that a curve $\xi:[0, T] \rightarrow \mathbb{R}^{N}$ is an integral trajectory of $F$ if

$$
\dot{\xi}(t) \in F(\xi(t)) \quad \text { for a.e. } t \in[0, T] \text {. }
$$

Definition 4.1 We say that a set $\Omega \subset \mathbb{R}^{N}$ is invariant for $F$ if $\Omega$ is a proper subset of $\mathbb{R}^{N}$ and for any integral curve $\xi$ of $F$ defined in some interval $[0, t]$ with $\xi(0) \in \Omega(\operatorname{resp} . \xi(t) \in \Omega)$, we have

$$
\xi(s) \in \Omega \quad \text { for any } s \in[0, t] .
$$

Our first result is:

Theorem 4.2 Any invariant set for the set-valued dynamic $\dot{\xi} \in F(\xi)$ in $\mathbb{R}^{N}$ has Lipschitz boundary.

Remark 4.3 The same statement holds clearly true for the dynamics given by $\dot{\xi} \in \mathcal{B}(V(\xi), 1)$, since the integral curves of the two problems are the same, up to change of parameter. The choice of $F$ fits our analysis better.

Remark 4.4 The above statement is nontrivial only if $H$ is noncoercive, or in other terms if $|V(x)| \geq 1$ for some $x$. If on the contrary $|V(x)|<1$ for any $x$ then $0 \in$ int $F(x)$ and any curve of $\mathbb{R}^{N}$ is an integral trajectory of $F$, up to change of parameter, so that invariant subsets cannot exist. Actually, we reach in our setting the conclusion that no periodic invariant set exist, but some mathematical effort is needed.

We need some preliminary material. The next assertion is easy to check.

Lemma 4.5 Given $\delta_{2}>\delta_{1}$ in $(0,1]$

$$
F_{\delta_{1}}(x) \subset F_{\delta_{2}}(y) \quad \text { for any } x \in \mathbb{R}^{N}, y \in \mathcal{B}\left(x, r_{V}\left(\delta_{1}, \delta_{2}\right)\right),
$$

with $r_{V}\left(\delta_{1}, \delta_{2}\right)=\frac{d_{2}-\delta_{1}}{L_{V}}$.

Lemma 4.6 Let $\Omega$ be an invariant set for $F$, then

$$
|V(x)| \geq 1 \quad \text { for any } x_{0} \in \partial \Omega .
$$

Proof Assume for purposes of contradiction that there is $x_{0} \in \partial \Omega$ with $\left|V\left(x_{0}\right)\right|<1$, then we find $r>0$ such that the same strict inequality holds true in $\mathcal{B}\left(x_{0}, r\right)$. This implies that

$$
0 \in \operatorname{int} F(x) \quad \text { for any } x \in \mathcal{B}\left(x_{0}, r\right) \text {. }
$$

Any curve lying in $\mathcal{B}\left(x_{0}, r\right)$ is therefore an integral curve of $F$, up to change of parameter. This is true in particular for a segment connecting a point in $\mathcal{B}\left(x_{0}, r\right) \cap \Omega$ to another point in $\mathcal{B}\left(x_{0}, r\right) \backslash \Omega$, which is in contrast with the invariance of $\Omega$. 
Lemma 4.7 Let $\Omega, x, \delta$ be an invariant set for $F$, a point in $\mathbb{R}^{N}$ and a constant in $(0,1)$, then

$$
\begin{array}{ll}
\left(y+\operatorname{int} F_{\delta}(x)\right) \cap \mathcal{B}\left(x, r_{V}(\delta, 1)\right) \subset \operatorname{int} \Omega & \text { for any } y \in \bar{\Omega} \cap U \\
\left(y-\operatorname{int} F_{\delta}(x)\right) \cap \mathcal{B}\left(x, r_{V}(\delta, 1)\right) \subset \operatorname{int} \Omega^{c} & \text { for any } y \in \overline{\Omega^{c}} \cap U .
\end{array}
$$

Proof To ease notations, we set $U=\mathcal{B}\left(x, r_{V}(\delta, 1)\right)$. First take $y \in \Omega \cap U$ and $q \in F_{\delta}(x)$ with $y+q \in U$, then the curve $y+t q, t \in[0,1]$, is an integral curve of $F$ in force of Lemma 4.5. This shows

$$
\left(y+F_{\delta}(x)\right) \cap U \subset \Omega
$$

and consequently

$$
\operatorname{int}\left(\left(y+F_{\delta}(x)\right) \cap U\right)=\left(y+\operatorname{int} F_{\delta}(x)\right) \cap U \subset \text { int } \Omega \quad \text { for any } y \in \Omega \cap U .
$$

Now consider $y \in \bar{\Omega} \cap U$ and denote by $y_{n}$ a sequence in $\Omega \cap U$ converging to $y$. If $q \in$ int $F_{\delta}(x)$ with $y+q \in U$, then

$$
q+y-y_{n} \in \operatorname{int} F_{\delta}(x) \quad \text { forn } \quad \text { sufficiently large }
$$

and consequently

$$
y+q=y_{n}+\left(q+y-y_{n}\right) \in\left(y_{n}+\operatorname{int} F_{\delta}(x)\right) \cap U .
$$

This proves

$$
\left(y+\operatorname{int} F_{\delta}(x)\right) \cap U \subset \bigcup_{n}\left(y_{n}+\operatorname{int} F_{\delta}(x)\right) \cap U,
$$

since $y_{n} \in \Omega \cap U$ for any $n$, all the sets in the right hand-side of the previous formula are contained in int $\Omega$ by (1). The first inclusion of the claim is then proved. The latter can be deduced by slightly adapting the same argument.

Proof of Theorem 4.2 Let $\Omega$ be an invariant set. Given $x \in \partial \Omega$, we select $\delta \in(0,1)$ and set $U=\mathcal{B}\left(x, r_{V}(\delta, 1)\right)$. We denote by proj the orthogonal projection on the space $x+V(x)^{\perp}$ and consider the set

$$
I:=\operatorname{proj}\left(\left(x+\operatorname{int} F_{\delta}(x)\right) \cap U\right) \cap \operatorname{proj}\left(\left(x-\operatorname{int} F_{\delta}(x)\right) \cap U\right),
$$

which is an open convex neighborhood of $x$.

Claim 1. For any $y \in I$ there is one and only one $\lambda=\lambda(y) \in \mathbb{R}$ such that $y+\lambda V(x) \in$ $\partial \Omega \cap U$.

Let $y$ be in $I$, by the very definition of $I$ there are $\lambda_{1}, \lambda_{2}$ with

$$
\begin{aligned}
& y+\lambda_{2} V(x) \in\left(x+\operatorname{int} F_{\delta}(x)\right) \cap U \\
& y+\lambda_{1} V(x) \in\left(x-\operatorname{int} F_{\delta}(x)\right) \cap U
\end{aligned}
$$

note that by Lemma $4.6|V(x)| \geq 1$, then $V(x)^{\perp}$ is a supporting hyperplane for both $x+F_{\delta}(x)$ and $x-F_{\delta}(x)$, and

$$
\begin{aligned}
& x+\operatorname{int} F_{\delta}(x) \subset\{z \mid(z-x) \cdot V(x)>0\} \\
& x-\operatorname{int} F_{\delta}(x) \subset\{z \mid(z-x) \cdot V(x)<0\} .
\end{aligned}
$$

This implies that $\lambda_{2}>0$ and $\lambda_{1}<0$. In addition, owing to the convexity of $U$ we have

$$
y+\lambda V(x) \in U \quad \text { for any } \lambda \in\left[\lambda_{1}, \lambda_{2}\right] .
$$


We deduce from Lemma 4.7

$$
\begin{aligned}
& y+\lambda_{2} V(x) \in \operatorname{int} \Omega \\
& y+\lambda_{1} V(x) \in \operatorname{int} \Omega^{c} .
\end{aligned}
$$

We set

$$
\begin{aligned}
\lambda^{\prime} & =\inf \left\{\lambda \in\left(\lambda_{1}, \lambda_{2}\right) \mid y+\lambda V(x) \in \operatorname{int} \Omega\right\} \\
\lambda^{\prime \prime} & =\sup \left\{\lambda \in\left(\lambda_{1}, \lambda_{2}\right) \mid y+\lambda V(x) \in \operatorname{int} \Omega^{c}\right\} .
\end{aligned}
$$

We proceed proving by contradiction that $\lambda^{\prime}=\lambda^{\prime \prime}$. In fact, if $\lambda^{\prime}>\lambda^{\prime \prime}$ then

$$
y+\lambda V(x) \in \partial \Omega \cap U \quad \text { for any } \lambda \in\left[\lambda^{\prime \prime}, \lambda^{\prime}\right],
$$

on the other side, we choose $\lambda$ in $\left[\lambda^{\prime \prime}, \lambda^{\prime}\right]$ so close to $\lambda^{\prime \prime}$ that

$$
\left(\lambda-\lambda^{\prime \prime}\right) V(x) \in \operatorname{int} F_{\delta}(x),
$$

and we deduce from (2) and Lemma 4.7

$$
\begin{aligned}
y+\lambda V(x) & =y+\lambda^{\prime \prime} V(x)+\left(\lambda-\lambda^{\prime \prime}\right) V(x) \\
& \in\left(\left(y+\lambda^{\prime \prime} V(x)\right)+\operatorname{int} F_{\delta}(x)\right) \cap U \subset \operatorname{int} \Omega,
\end{aligned}
$$

which is impossible. If instead $\lambda^{\prime}<\lambda^{\prime \prime}$, then we set

$$
\lambda_{0}=\sup \left\{\lambda \in\left[\lambda^{\prime}, \lambda^{\prime \prime}\right] \mid y+\lambda V(x) \in \operatorname{int} \Omega\right\} .
$$

We find $\lambda<\lambda_{0}$ close to $\lambda_{0}$ with

$$
\begin{gathered}
y+\lambda V(x)=\operatorname{int} \Omega \\
\left(\lambda_{0}-\lambda\right) V(x) \in \operatorname{int} F_{\delta}(x)
\end{gathered}
$$

and

$$
\begin{aligned}
y+\lambda_{0} V(x) & =y+\lambda V(x)+\left(\lambda_{0}-\lambda\right) V(x) \\
& \in\left((y+\lambda V(x))+\operatorname{int} F_{\delta}(x)\right) \cap U \subset \operatorname{int} \Omega,
\end{aligned}
$$

contrast with the definition of $\lambda_{0}$. We have in conclusion established by contradiction that $\lambda^{\prime}=\lambda^{\prime \prime}$.

Claim 2. The function

$$
y \mapsto\{\lambda \mid y+\lambda V(x) \in \partial \Omega\}
$$

from I to $\mathbb{R}$ is Lipschitz-continuous.

The function, that we denote by $\psi$, is univocally defined by the previous claim, and is continuous by its very definition and the fact that $\partial \Omega$ is closed. By squeezing a bit $I$, we can in addition suppose without loosing generality that $\psi$ is uniformly continuous so that we can determine $\varepsilon$ with

$$
\left|y_{1}-y_{2}\right|<\varepsilon \Rightarrow\left|\psi\left(y_{1}\right)-\psi\left(y_{2}\right)\right|<1 .
$$

We pick such a pair $y_{1}, y_{2}$, and assume that $\psi\left(y_{1}\right)>\psi\left(y_{2}\right)$, therefore

$$
\mathcal{B}\left(\left(\psi\left(y_{1}\right)-\psi\left(y_{2}\right)\right) V(x),\left(\psi\left(y_{1}\right)-\psi\left(y_{2}\right)\right) \delta\right) \subset \text { int } F_{\delta}(x)
$$

and consequently by Lemma 4.7

$$
\left(\left(y_{2}+\psi\left(y_{2}\right) V(x)\right)+\mathcal{B}\left(\left(\psi\left(y_{1}\right)-\psi\left(y_{2}\right)\right) V(x),\left(\psi\left(y_{1}\right)-\psi\left(y_{2}\right)\right) \delta\right)\right) \cap U \subset \text { int } \Omega .
$$


Since $y_{1}+\psi\left(y_{1}\right) V(x) \in \partial \Omega \cap U$, we must therefore have

$$
\begin{aligned}
\left|y_{2}-y_{1}\right| & =\left|y_{1}+\psi\left(y_{1}\right) V(x)-y_{2}-\psi\left(y_{2}\right) V(x)-\left(\psi\left(y_{1}\right)-\psi\left(y_{2}\right)\right) V(x)\right| \\
& \geq\left(\psi\left(y_{1}\right)-\psi\left(y_{2}\right)\right) \delta
\end{aligned}
$$

This shows the claim. To conclude, it is enough to notice, see Definition 2.1, that by Lemma 4.7 the set

$$
\{y+\lambda \psi(y) \mid y \in I, \lambda>\psi(y)\}
$$

is contained in int $\Omega$.

Definition 4.8 We say that a subset $A \subset \mathbb{R}^{N}$ is periodic if

$$
x \in A \Rightarrow x+z \in A \quad \text { for anyz } \in \mathbb{Z}^{N} .
$$

The main consequence of the Theorem 4.2 is:

Theorem 4.9 The dynamics given by $F$ does not have any invariant periodic subset.

Remark 4.10 Note that invariant non periodic sets for $F$ could exist in our setting. It is enough to consider for instance $V(x)$ constant.

We preliminarily give two lemmata. We define for $x \in \mathbb{R}^{N}$

$$
Z^{\infty}(x)=\{p \mid H(x, p) \leq 0\},
$$

It is apparent that $Z^{\infty}(x)$ is the recession cone of the sublevels $\{p \mid H(x, p) \leq a\}$ for $a>0$.

\section{Lemma 4.11}

$$
F(x)^{-}=Z^{\infty}(x) \quad \text { for any } x \in \mathbb{R}^{N} .
$$

Proof An element of $F(x)$ can be written in the form

$$
\lambda(V(x)+q) \quad \text { for } 0 \leq \lambda \leq 1,|q| \leq 1 .
$$

Let $p \in Z^{\infty}(x)$, then

$$
p \cdot(V(x)+q) \leq-|p|+p \cdot q \leq 0,
$$

which shows that

$$
Z^{\infty}(x) \subset F(x)^{-} .
$$

Conversely, let $p \in F(x)^{-}$, then $V(x)+\frac{p}{|p|} \in F(x)$, and so

$$
0 \geq p \cdot\left(V(x)+\frac{p}{|p|}\right)=H(x, p),
$$

which implies that $p \in Z^{\infty}(x)$. This concludes the proof.

Lemma 4.12 Let $\Omega \subset \mathbb{R}^{N}$ be an invariant set for $F$. Then

$$
n_{\Omega}(x) \cdot V(x) \leq-1 \quad \text { for } \mathcal{H}^{N-1} \quad \text { a.e. } x \in \partial \Omega,
$$

where $n_{\Omega}$ denotes the outer unit normal to $\Omega$ and $\mathcal{H}^{N-1}$ is the $(N-1)$-dimensional Hausdorff measure. 
Proof Recall that $\Omega$ has Lipschitz boundary by Theorem 4.2. Let $x$ be a point of $\partial \Omega$ where there exists the outer normal $n_{\Omega}(x)$, see Remark 2.2. We recall that by Lemma $4.6|V(x)| \geq 1$. By Lemma 4.7, we can find for any $\delta \in(0,1)$ an open ball $U$ centered at $x$ with

$$
\left(x+\text { int } F_{\delta}(x)\right) \cap U \subset \text { int } \Omega,
$$

which implies that $n_{\Omega}(x)$ is normal to $x+F_{\delta}(x)$ at $x$ and consequently $n_{\Omega}(x) \in F_{\delta}(x)^{-}$. Therefore, by Lemma 4.11

$$
n_{\Omega}(x) \in \bigcap_{\delta \in(0,1)} F_{\delta}(x)^{-}=F(x)^{-}=Z^{\infty}(x),
$$

or, in other terms

$$
0 \geq H\left(x, n_{\Omega}(x)\right) \geq 1+n_{\Omega}(x) \cdot V(x) .
$$

This proves (3).

Proof of Theorem 4.9 Assume for purposes of contradiction that there exists a periodic invariant set for $F$. We denote by $\Theta$ its projection on $\mathbb{T}^{N}$, by Theorem $4.2 \Theta$ possess Lipschitz boundary, so that we are in position to apply divergence Theorem and, owing to Lemma 4.12, we get

$$
\mathcal{H}^{N-1}(\partial \Theta) \leq \int_{\partial \Theta}-n_{\Theta} \cdot V d \mathcal{H}^{N-1}=\left|\int_{\Theta} \operatorname{div} V d x\right| \leq\|\operatorname{div} V\|_{L^{N}\left(\mathbb{T}^{N}\right)}|\Theta|^{1-1 / N} .
$$

Exploiting assumption (A2) on $\operatorname{div} V$ we deduce

$$
\mathcal{H}^{N-1}(\partial \Theta)<\frac{1}{\chi}|\Theta|^{1-1 / N}
$$

which is contrast with the isoperimetric inequality, see Sect. 2.

\section{Intrinsic distances}

We associate to the 1-sublevels of $H$ an intrinsic distance. The first step is to define for any $x, q$ in $\mathbb{R}^{N}$

$$
\begin{aligned}
Z(x) & =\{p \mid H(x, p) \leq 1\} \\
\sigma(x, q) & =\sup \{p \cdot q \mid p \in Z(x)\}
\end{aligned}
$$

The set-valued function $Z$ is closed convex valued and 0 is an interior point of $Z(x)$ for any $x$. This implies that $\sigma(x, q)$ is strictly positive when $q \neq 0$.

For $|V(x)|$ increasing, the sublevel $Z(x)$ shrinks in the direction of $V(x)$ and stretches in the opposite one, assuming more or less the shape of a water drop, till $|V(x)|$ reaches the threshold value of 1 , where it breaks apart and the unbounded recession cone $Z^{\infty}(x)$ pops up as a subset. Accordingly, the support function $\sigma(x, \cdot)$ becomes infinite at some $q$.

The next result is straightforward, we provide a proof in the Appendix for completeness.

Proposition 5.1 The function $(x, q) \mapsto \sigma(x, q)$ from $\mathbb{R}^{n} \times \mathbb{R}^{N}$ to $\mathbb{R}^{+} \cup\{+\infty\}$ is lower semicontinuous. In addition $q \mapsto \sigma(x, q)$ is convex positively homogeneous, for any fixed $x$.

The next result puts in relation the present construction and $F$. 
Lemma 5.2 We have

$$
\sigma(x, q) \leq 1 \quad \text { for any } x \in \mathbb{R}^{N}, q \in F(x) .
$$

Proof Given

$$
q=V(x)+\bar{q} \quad \text { with } \bar{q} \quad \text { unit vector }
$$

$p \in Z(x)$, we have

$$
p \cdot q=p \cdot V(x)+p \cdot \bar{q} \leq p \cdot V(x)+|p| \leq 1,
$$

which shows that $\sigma(x, q) \leq 1$. Since $F(x)$ is the convex hull of vectors of the form (4) plus 0 , we get the assertion.

For any curve $\xi:[0, T] \rightarrow \mathbb{R}^{N}$ we define the intrinsic length functional $\ell_{V}$ by

$$
\ell_{V}(\xi)=\int_{0}^{T} \sigma(\xi, \dot{\xi}) d t
$$

Notice that the above integral is invariant for orientation-preserving change of parameter, as an intrinsic length should be. It is in addition positive, and can be infinite. The corresponding path-metric $S_{1}$ is given by

$$
S_{1}(x, y)=\inf \left\{\ell_{V}(\xi) \mid \xi \text { joining } x \text { to } y\right\},
$$

for any $x, y$ in $\mathbb{R}^{N}$. The (semi)distance $S_{1}$ is strictly positive whenever the two arguments are distinct, and can be infinite. Note that $S_{1}(x, x)=0$ for any $x$, in addition $S_{1}$ enjoys the triangle property, but it is not in general symmetric.

We provide a periodic version of $S_{1}$ via the formula

$$
\bar{S}_{1}(x, y)=\inf \left\{S_{1}\left(x+z, y+z^{\prime}\right) \mid z, z^{\prime} \in \mathbb{Z}^{N}\right\},
$$

Due to the periodic character of $H$, we also have

$$
\bar{S}_{1}(x, y)=\inf \left\{S_{1}(x+z, y) \mid z \in \mathbb{Z}^{N}\right\}=\inf \left\{S_{1}(x, y+z) \mid z \in \mathbb{Z}^{N}\right\} .
$$

The following lemma will be used in Sect. 6 .

Lemma 5.3 Let $P$ be an element of $\mathbb{R}^{N}, \delta<1$. For any $x \in \mathbb{R}^{N}$ there are two open sets $U_{x}$, $W_{x}$ with $x \in \overline{U_{x}} \cap \overline{W_{x}}$ such that

$$
\begin{array}{ll}
S_{1}(x, y)+P \cdot(x-y)<1+|P| r_{V}(\delta, 1) & \text { for any } y \in U_{x} \\
S_{1}(y, x)+P \cdot(y-x)<1+|P| r_{V}(\delta, 1) & \text { for anyy } \in W_{x} .
\end{array}
$$

In addition both families $\left\{U_{x}\right\},\left\{W_{x}\right\}$, for $x \in \mathbb{R}^{N}$, make up an open covering of the whole space.

Proof Given $x \in \mathbb{R}^{N}$, we have by Lemma 4.5 that

$$
F_{\delta}(x) \subset F(y) \quad \text { for any } y \in \mathcal{B}\left(x, r_{V}(\delta, 1)\right) .
$$

We set

$$
\begin{aligned}
U_{x} & =\left(x+\operatorname{int} F_{\delta}(x)\right) \cap \mathcal{B}\left(x, r_{V}(\delta, 1)\right) \\
W_{x} & =\left(x-\operatorname{int} F_{\delta}(x)\right) \cap \mathcal{B}\left(x, r_{V}(\delta, 1)\right),
\end{aligned}
$$


if $y \in U_{x}$ then $y-x \in \operatorname{int} F_{\delta}(x)$ and $x+t(y-x) \in \mathcal{B}\left(x, r_{V}(\delta, 1)\right)$, for $t \in[0,1]$, we therefore derive from (9) and Lemma 5.2

$$
\sigma(x+t(y-x), y-x)<1 \quad \text { for } t \in[0,1] .
$$

This implies that the intrinsic length of the segment $x+t(y-x), t \in[0,1]$ is less than 1 showing (7). Item (8) can be proven following the same lines.

We proceed proving that for any $x \in \mathbb{R}^{N}$ there is $y$ with $x \in U_{y}$. We select $\beta>0$ with

$$
\beta<\min \left\{1, \frac{1-\delta}{M_{V} L_{V}}\right\},
$$

and set

$$
y=x-\beta V(x) .
$$

We get

$$
|y-x|=\beta|V(x)| \leq \beta M_{V}<\frac{1-\delta}{L_{V}}=r_{V}(\delta, 1)
$$

and

$$
|x-y-\beta V(y)|=\beta|V(x)-V(y)|<\beta .
$$

The above inequalities show that $x \in U_{y}$. Adapting the above argument, we also find $z$ with $x \in W_{z}$. This concludes the proof.

Remark 5.4 Slightly adapting the above construction, a distance, denoted by $S_{a}, \bar{S}_{a}$ can be defined starting from the sublevels $\{p \mid H(x, p) \leq a\}$, for any $a>0$. Due to the positive homogeneity of $H$ in $p$, we have for any $x, y$ in $\mathbb{R}^{N}$

$$
\begin{aligned}
& S_{a}(x, y)=a S_{1}(x, y) \\
& \bar{S}_{a}(x, y)=a \bar{S}_{1}(x, y)
\end{aligned}
$$

Furthermore, it is easy to check that the intrinsic periodic distance corresponding to the level 0 is identically vanishing, thanks to Theorem 4.9 , while $\bar{S}_{a}$ is identically equal to $-\infty$ for $a<0$.

\section{Homogenization}

We consider the family of equations

$$
H(x, D u+P)=a \quad a \in \mathbb{R},
$$

with $P \in \mathbb{R}^{N}$ fixed, and define the effective Hamiltonian $\bar{H}$ as

$$
\bar{H}(P)=\inf \{a \mid(10) \text { admits an usc bounded periodic subsolution }\} .
$$

The effective Hamiltonian is the one appearing in the limit equation $(\overline{\mathrm{HJ}})$ of the homogenization procedure.

Lemma 6.1 The effective Hamiltonian $\bar{H}$ is convex positively homogeneous and, in addition

$$
\min _{x \in \mathbb{R}^{N}} H(x, P) \leq \bar{H}(P) \leq \max _{x \in \mathbb{R}^{N}} H(x, P) \quad \text { for any } P \in \mathbb{R}^{N}
$$


Proof The effective Hamiltonian $\bar{H}$ directly inherits convexity and positive homogeneity from $H$. If $a \geq \max _{x \in \mathbb{R}^{N}} H(x, P)$ then any constant function is a subsolution to (10), which gives the rightmost inequality in the statement.

Next, let $u$ be a bounded periodic usc subsolution to (10). We denote by $x_{0}$ a maximizer of it in $\mathbb{R}^{N}$. Then $H\left(x_{0}, P\right) \leq a$ and so $\min _{x \in \mathbb{R}^{N}} H(x, P) \leq a$. This completes the proof.

We proceed fixing $P$. Following the same procedure as in Sect. 5, we see that the intrinsic distance related to the sublevel $H(x, p+P) \leq a$ is

$$
D_{a}(x, y)=S_{a}(x, y)+P \cdot(x-y),
$$

and the periodic version is

$$
\begin{aligned}
\bar{D}_{a}(x, y) & =\inf _{z, z^{\prime} \in \mathbb{Z}^{N}}\left\{S_{a}\left(x+z, y+z^{\prime}\right)+P \cdot\left(x+z-y-z^{\prime}\right)\right\} \\
& =\inf _{z \in \mathbb{Z}^{N}}\left\{S_{a}(x, y+z)+P \cdot(x-y-z)\right\}
\end{aligned}
$$

Remark 6.2 Note that $\bar{D}_{a}$ could be finite even for $a<0$, despite the fact that $\bar{S}_{a}$ is identically equal to $-\infty$. This is related to the problem of the possible coercivity of $\bar{H}$. We do not treat this issue here, see [3,9].

The next result is a relevant consequence of Theorem 4.9.

Theorem 6.3 The distance $\bar{D}_{1}$ is bounded from above in $\mathbb{R}^{N} \times \mathbb{R}^{N}$.

Proof We first show that $\bar{D}_{1}$ is bounded from above in $\mathbb{R}^{N} \times \mathbb{R}^{N}$, provided it is finite. Given $\delta<1$, we consider the families of open sets $\left\{U_{x}\right\},\left\{W_{x}\right\}$, for $x \in \bar{Q}_{1}$, introduced in Lemma 5.3 , and extract finite subfamilies $\left\{U_{i}\right\}, i=1, \ldots, M_{1},\left\{W_{j}\right\}, j=1, \ldots, M_{2}$, respectively, corresponding to points $x_{i}, \bar{x}_{j}$, covering $\bar{Q}_{1}$.

Due to Lemma 5.3, we have that for any $x \in \bar{Q}_{1}$ there are indices $i_{0}, j_{0}$ with

$$
\bar{D}_{1}\left(x_{i_{0}}, x\right) \leq 1+|P| r_{V}(\delta, 1) \quad \text { and } \quad \bar{D}_{1}\left(x, \bar{x}_{j_{0}}\right) \leq 1+|P| r_{V}(\delta, 1) .
$$

Since we are supposing that $\bar{D}_{1}$ is finite, we have

$$
\bar{D}_{1}\left(\bar{x}_{j}, x_{i}\right) \leq \alpha \quad \text { for } i=1, \ldots, M_{1}, j=1, \ldots, M_{2}, \quad \text { some } \alpha>0 .
$$

We take any pair of points $y, z$ in $\bar{Q}_{1}$ and assume that (14) holds true with $z$ and $y$ in place of $x$, respectively. Then

$$
\bar{D}_{1}(y, z) \leq \bar{D}_{1}\left(y, \bar{x}_{j_{0}}\right)+\bar{D}_{1}\left(\bar{x}_{j_{0}}, x_{i_{0}}\right)+\bar{D}_{1}\left(x_{i_{0}}, z\right) \leq 2+2|P| r_{V}(\delta, 1)+\alpha,
$$

showing the claim.

We proceed proving that since periodic invariant sets for $F$ do not exist in force of Theorem 4.9 , then $\bar{D}_{1}$ is actually finite in $\mathbb{R}^{N} \times \mathbb{R}^{N}$. It is sufficient to show that, for any given $y_{0} \in \mathbb{R}^{N}$, the set

$$
\Omega=\left\{x \in \mathbb{R}^{N} \mid \bar{D}_{1}\left(y_{0}, x\right)<+\infty\right\}
$$

is periodic invariant for $F$. This in fact implies that $\Omega=\mathbb{R}^{N}$, from which we in turn deduce, by the arbitrariness of $y$, that $\bar{D}_{1}$ is finite.

It is clear by its very definition that $\Omega$ is periodic. To show the invariance, we take $x_{0} \in \Omega$ and consider an integral curve $\xi$ of $F$ defined in $[0, T)$, for some $T \in \mathbb{R}^{+} \cup\{+\infty\}$, issued 
from $x_{0}$. Then we have by Lemma 5.2

$$
\begin{aligned}
\bar{D}_{1}\left(y_{0}, \xi(t)\right) & \leq \bar{D}_{1}\left(y_{0}, x_{0}\right)+\ell_{V}\left(\left.\xi\right|_{[0, t]}\right)+P \cdot\left(x_{0}-\xi(t)\right) \\
& \leq \bar{D}_{1}\left(y_{0}, x_{0}\right)+t+P \cdot\left(x_{0}-\xi(t)\right) \quad \text { for any } t \in[0, T) .
\end{aligned}
$$

Therefore the entire curve $\xi$ is contained in $\Omega$, as claimed.

We proceed defining a sequence of coercive Hamiltonians approximating $H$. We set for $k \in \mathbb{N}$

$$
H_{k}(x, p)=\max \{H(x, p),-k\}+f_{k}(p)
$$

where

$$
f_{k}(p)=\left\{\begin{array}{cc}
0 & \text { if }|p|<k \\
|p|-k & \text { if }|p| \geq k
\end{array}\right.
$$

and define the related intrinsic distances $S_{a}^{k}, \bar{S}_{a}^{k}, D_{a}^{k}, \bar{D}_{a}^{k}$ adapting formulae (5), (6), (12), (13).

The Hamiltonians $H_{k}$ are continuous, periodic in the state and convex in the momentum variable. They in addition satisfy:

- the sequence $H_{k}(x, p)$ is nonincreasing for any $(x, p)$;

- $H_{k}$ converges to $H$ locally uniformly in $\mathbb{R}^{N} \times \mathbb{R}^{N}$ by Dini's monotone convergence theorem.

The previous properties clearly imply that

$$
H(x, p) \leq H_{k}(x, p) \quad \text { for any } k, x, p .
$$

The effective Hamiltonians $\bar{H}_{k}$, corresponding to $H_{k}$, are defined as in (11) with obvious adaptations. Notice that, being $H_{k}$ coercive, any periodic subsolution to (10), with $H_{k}$ in place of $H$, is automatically Lipschitz-continuous. In addition the critical equation $H_{k}(x, P+$ $D u)=\bar{H}_{k}(P)$ possess solutions. We also have that the $\bar{H}_{k}$ are convex coercive.

The main result of the section is:

Theorem $6.4-$ For any $P \in \mathbb{R}^{N}, \lim _{k} \bar{H}_{k}(P)=\bar{H}(P)$;

- there exist bounded periodic usc subsolutions and lsc supersolutions to the equation $H(x, D u+P)=\bar{H}(P)$.

Note that, in absence of coerciveness of $H$, the convergence of $\bar{H}_{k}$ to $\bar{H}$ is not a straightforward consequence of the fact that $H_{k} \rightarrow H$ locally uniformly.

One half of the previous theorem is actually easy and is indeed a direct consequence of the properties of $H_{k}$. In fact, if $u$ is a subsolution to $H_{k}(x, D u+P)=a$, for some a, then it enjoys the same property with respect to $H_{j}(x, D u+P)=a$, whenever $j<k$, and with respect to (10). This actually shows:

Lemma 6.5 The sequence $\bar{H}_{k}(P)$ is nonincreasing and

$$
\bar{H}_{k}(P) \geq \bar{H}(P) \quad \text { for anyk. }
$$

Remark 6.6 Since the matter is somehow intricate, let us summarize the different monotonicity arising in the interplay between the $H_{k}$ and $H$.

- $H_{k}(x, p)$ is nonincreasing and $H_{k}(x, p) \rightarrow H(x, p)$, for any $(x, p)$; 
- $S_{a}^{k}(x, y)$ is nondecreasing and $S_{a}^{k}(x, y) \leq S_{a}(x, y)$, for any $x, y, a$;

- $\bar{H}_{k}(P)$ is nonincreasing and the possible convergence of $\bar{H}_{k}(P)$ to $\bar{H}(P)$ is the subject we are presently investigating.

We need some preliminary material for the proof of Theorem 6.4.

Lemma 6.7 Let $a \geq \bar{H}_{1}(P)$, then $\bar{D}_{a}$ is bounded in $\mathbb{R}^{N} \times \mathbb{R}^{N}$.

Proof We start proving that it is bounded from above. If $a \leq 0$ then $\bar{D}_{a} \leq \bar{D}_{1}$, if $a>0$ then

$$
\bar{D}_{a}(x, y)=a \inf _{z \in \mathbb{Z}^{N}}\left\{S_{1}(x, y)+P / a \cdot(x-y)\right\} .
$$

It is then enough to prove that $\bar{D}_{1}$ is bounded from above. This property actually comes from Theorem 6.3. To see that $\bar{D}_{a}$ is also bounded from below, we recall that there is a periodic solution $u$ of

$$
H_{1}(x, D v+P)=\bar{H}_{1}(P) .
$$

Then $u$ is a subsolution to

$$
H(x, D v+P)=a
$$

and, since $u$ is Lipschitz continuous for the coerciveness of $H_{1}$, we have

$$
u(x)-u(y) \leq \bar{D}_{a}(y, x) \quad \text { for any } x, y
$$

showing the claim.

We derive:

Lemma 6.8 The family of the distances $\left(\bar{D}_{H_{k}(P)}^{k}\right)_{k}$ are equibounded in $\mathbb{R}^{N} \times \mathbb{R}^{N}$.

Proof We start proving the boundedness from above. Taking into account Remark 6.6, we have

$$
\bar{D} \bar{H}_{k(P)}^{k}(x, y) \leq \bar{D}_{\bar{H}_{1}(P)}^{k}(x, y) \leq \bar{D}_{\bar{H}_{1}(P)}(x, y) .
$$

To get the converse estimate, we first notice that

$$
0=\bar{D}_{\bar{H}_{k}(P)}^{k}(x, x)=\inf _{z \in \mathbb{Z}^{N}}\left\{S_{\bar{H}_{k}(P)}^{k}(x, x+z)-P \cdot z\right\}
$$

for any $x \in \mathbb{R}^{N}$. We consider a pair of points $x, y$ in $\mathbb{R}^{N}$. We select $\bar{z} \in \mathbb{Z}^{N}$ in such a way that

$$
S_{\bar{H}_{1}(P)}(y, x+\bar{z})+P \cdot(y-x-\bar{z}) \leq \bar{D}_{\bar{H}_{1}(P)}(y, x)+1 .
$$


We compute taking into account (16), Remark 6.6 and Lemma 6.7

$$
\begin{aligned}
\bar{D} \bar{H}_{(P)}^{k}(x, y) & \left.=\inf _{z \in \mathbb{Z}^{N}}\left\{S_{\bar{H}_{k}(P)}^{k}(x+z, y)\right)+P \cdot(x+z-y)\right\} \\
& \geq \inf _{z \in \mathbb{Z}^{N}}\left\{S_{\bar{H}_{k}(P)}^{k}(x+z, x+\bar{z})-S_{\bar{H}_{k}(P)}^{k}(y, x+\bar{z})\right. \\
& +P \cdot(z-\bar{z})-P \cdot(y-x-\bar{z})\} \\
& \geq \inf _{z \in \mathbb{Z}^{N}}\left\{S_{\bar{H}_{k}(P)}^{k}(x+z, x+\bar{z})+P \cdot(z-\bar{z})\right\} \\
& +P \cdot(x+\bar{z}-y)-S_{\bar{H}_{k}(P)}^{k}(y, x+\bar{z}) \\
& \geq P \cdot(x+\bar{z}-y)-S_{\bar{H}_{k}(P)}(y, x+\bar{z}) \\
& \geq P \cdot(x+\bar{z}-y)-S_{\bar{H}_{1}(P)}(y, x+\bar{z}) \geq-\bar{D}_{\bar{H}_{1}(P)}(y, x)-1 .
\end{aligned}
$$

This concludes the proof.

Lemma 6.9 If for a given a there is a bounded periodic usc subsolution and a bounded periodic lsc supersolution of (10), then $a=\bar{H}(P)$.

The proof is basically the same as in coercive case, with some more precaution. We present it in the Appendix.

We need the following result, see for instance [6].

Proposition 6.10 For any $k$, any $y$ in the Aubry set related to $H_{k}$, the function $x \mapsto$ $\bar{D} \frac{k}{\bar{H}_{k}(P)}(y, x)$ is a periodic Lipschitz continuous solution of $H_{k}(x, P+D u)=\bar{H}_{k}(P)$.

Proof of Theorem 6.4 We know from Proposition 6.10, that we can select, for any $k, y_{k}$ in such a way that $v_{k}:=\bar{D}_{\bar{H}_{k}(P)}^{k}\left(y_{k}, \cdot\right)$ is solution to

$$
H_{k}(x, D u+P)=\bar{H}_{k}(P) .
$$

Since the $v_{k}$ are periodic, equibounded by Lemma 6.8, the functions

$$
v:=\liminf \operatorname{in}_{k} \quad \text { and } \quad w:=\lim \sup ^{\#} v_{k}
$$

are bounded periodic and, taking into account the local uniform convergence of $H_{k}$ to $H$ plus standard stability properties of viscosity solutions theory, are indeed lsc supersolution and usc subsolution, respectively, to

$$
H(x, D u+P)=\lim _{k} \bar{H}_{k}(P) .
$$

This implies by Lemma 6.9 that $\bar{H}(P)=\lim _{k} \bar{H}_{k}(P)$. The assertion then follows.

Exploiting Theorem 6.4, we get the homogenization result in a quite standard way. We state it below and provide the proof in the Appendix.

Theorem 6.11 The solutions to $\left(H J_{\varepsilon}\right)$ locally uniformly converge to the solution of $(\overline{\mathrm{HJ}})$, where $\bar{H}$ is the effective Hamiltonian defined in (11).

Funding Open access funding provided by Università degli Studi di Roma La Sapienza within the CRUICARE Agreement. 
Open Access This article is licensed under a Creative Commons Attribution 4.0 International License, which permits use, sharing, adaptation, distribution and reproduction in any medium or format, as long as you give appropriate credit to the original author(s) and the source, provide a link to the Creative Commons licence, and indicate if changes were made. The images or other third party material in this article are included in the article's Creative Commons licence, unless indicated otherwise in a credit line to the material. If material is not included in the article's Creative Commons licence and your intended use is not permitted by statutory regulation or exceeds the permitted use, you will need to obtain permission directly from the copyright holder. To view a copy of this licence, visit http://creativecommons.org/licenses/by/4.0/.

\section{A some proofs}

Proof of Proposition 5.1 Let us consider $\left(x_{0}, q_{0}\right)$ and an approximating sequence $\left(x_{n}, q_{n}\right)$. First assume $\sigma\left(x_{0}, q_{0}\right)<+\infty$ and pick $p_{0} \in Z\left(x_{0}\right)$ with $\sigma\left(x_{0}, q_{0}\right) \leq p_{0} \cdot q_{0}+\delta$, for some positive $\delta$. Taking into account that the interior of $Z\left(x_{n}\right)$ is nonempty, there is a sequence $p_{n} \in Z\left(x_{n}\right)$ converging to $p_{0}$. We find

$$
\liminf _{n} \sigma\left(x_{n}, q_{n}\right) \geq \lim _{n} p_{n} \cdot q_{n}=p_{0} \cdot q_{0} \geq \sigma\left(x_{0}, q_{0}\right)-\delta,
$$

and the claimed semicontinuity follows since $\delta$ has been arbitrarily chosen .

If $\sigma\left(x_{0}, q_{0}\right)=+\infty$, then taking any $\beta>0$, we find $p_{0} \in Z\left(x_{0}\right)$ with $p_{0} \cdot q_{0} \geq \beta$, then

$$
\liminf _{n} \sigma\left(x_{n}, q_{n}\right) \geq \lim _{n} p_{0} \cdot q_{n}=p_{0} \cdot q_{0} \geq \beta,
$$

which implies $\lim _{n} \sigma\left(x_{n}, q_{n}\right)=+\infty$.

The claimed positive homogeneity and subadditivity are immediate.

Proof of Lemma 6.9 The argument is by contradiction. Let $a$ be such that (10) has supersolution and subsolution as specified in the statement, denoted by $v, u$, respectively. If $a>\bar{H}(P)$, then we can select $b<a$ with

$$
H(x, D u+P) \leq b \quad \text { in the viscosity sense. }
$$

We denote, for any $\delta>0$, by $u^{\delta}$ the sup-convolutions of $u$. By basic properties of it, we know that

$$
H\left(y, \frac{x-y}{\delta}\right) \leq b
$$

for any $\delta$, any $x \in \mathbb{R}^{N}, y u^{\delta}$-optimal to $x$. We proceed considering a sequence

$$
x_{\delta} \in \arg \min _{\mathbb{R}^{N}}\left\{v-u^{\delta}\right\},
$$

note that such minimizers do exist because $v$ is lsc, $u^{\delta}$ usc, and both $v$ and $u^{\delta}$ are periodic. We denote, for any $\delta$, by $y_{\delta}$ an element $u^{\delta}$-optimal for $x_{\delta}$, we have

$$
\lim _{\delta} \frac{\left|x_{\delta}-y_{\delta}\right|^{2}}{\delta}=0
$$

Consequently, taking into account the inequality

$$
\left|H\left(x_{\delta}, \frac{x_{\delta}-y_{\delta}}{\delta}\right)-H\left(y_{\delta}, \frac{x_{\delta}-y_{\delta}}{\delta}\right)\right| \leq L_{V} \frac{\left|x_{\delta}-y_{\delta}\right|^{2}}{\delta}
$$


and that $b<a$, we conclude that

$$
H\left(x_{\delta}, \frac{x_{\delta}-y_{\delta}}{\delta}\right)<a,
$$

for $\delta$ small enough. On the other side, keeping in mind that $\frac{x_{\delta}-y_{\delta}}{\delta} \in D^{-} u^{\delta}\left(x_{\delta}\right)$ and that $u^{\delta}$ is subtangent to $v$ at $x_{\delta}$, we get

$$
H\left(x_{\delta}, \frac{x_{\delta}-y_{\delta}}{\delta}\right) \geq a \quad \text { for any } \delta,
$$

which contradicts (19).

Proof of Theorem 6.11 Let $u_{\varepsilon}$ the solutions to $\left(\mathrm{HJ}_{\varepsilon}\right)$, they are equibounded by (A3). We set

$$
v=\liminf \# u_{\varepsilon} \quad \text { and } \quad u=\lim \sup ^{\#} u_{\varepsilon} .
$$

As already pointed out in the Introduction, it is standard to show that $v$ is a supersolution of $(\overline{\mathrm{HJ}})$.

We focus on proving that $u$ is subsolution to $(\overline{\mathrm{HJ}})$. We consider $\left(x_{0}, t_{0}\right) \in \mathbb{R}^{N} \times(0,+\infty)$ and a $C^{1}$ strict supertangent $\psi$ to $u$ at $\left(x_{0}, t_{0}\right)$. We denote by $w$ a bounded periodic lsc supersolution to the problem

$$
H\left(x, D v+D\left(\psi\left(x_{0}, t_{0}\right)\right)\right)=\bar{H}\left(D\left(\psi\left(x_{0}, t_{0}\right)\right)\right) .
$$

We have that $\varepsilon w(x / \varepsilon)$ is supersolution to

$$
H\left(x / \varepsilon, D v+D\left(\psi\left(x_{0}, t_{0}\right)\right)\right)=\bar{H}\left(D\left(\psi\left(x_{0}, t_{0}\right)\right)\right) .
$$

The point $\left(x_{0}, t_{0}\right)$ is the unique maximizer of $u-\psi$ in $\bar{U}$, for a suitable choice of an open neighborhood $U$ of $\left(x_{0}, t_{0}\right)$ in $\mathbb{R}^{N} \times(0,+\infty)$. We proceed considering the functions

$$
u_{\varepsilon}(x, t)-\psi(x, t)-\varepsilon w_{\delta}(x / \varepsilon)
$$

where $w_{\delta}$ stands for the $\delta$ inf-convolution of $w$ for $\delta>0$. We have that the quantity $\varepsilon w_{\delta}(x / \varepsilon)$ converges uniformly to 0 as $\varepsilon \rightarrow 0$, uniformly with respect to $\delta$. Owing to the stability properties of maximizers, we deduce that a sequence of maximizers of (21) denoted by $\left(x_{\varepsilon}^{\delta}, t_{\varepsilon}^{\delta}\right)$, belongs to $U$ for $\varepsilon$ small enough and

$$
\left(x_{\varepsilon}^{\delta}, t_{\varepsilon}^{\delta}\right) \rightarrow\left(x_{0}, t_{0}\right) \quad \text { as } \varepsilon \rightarrow 0, \text { uniformly in } \delta .
$$

Since

$$
\left(\psi_{t}\left(x_{\varepsilon}^{\delta}, t_{\varepsilon}^{\delta}\right), D \psi\left(x_{\varepsilon}^{\delta}, t_{\varepsilon}^{\delta}\right)+p_{\varepsilon}^{\delta}\right) \in D^{+} u_{\varepsilon}\left(x_{\varepsilon}^{\delta}, t_{\varepsilon}^{\delta}\right)
$$

for any $p_{\varepsilon}^{\delta} \in \partial w_{\delta}\left(x_{\varepsilon}^{\delta}\right)=D^{+} w_{\delta}\left(x_{\varepsilon}^{\delta}\right)$, and $u_{\varepsilon}$ is subsolution to $\left(\mathrm{HJ}_{\varepsilon}\right)$, we derive

$$
\psi_{t}\left(x_{\varepsilon}^{\delta}, t_{\varepsilon}^{\delta}\right)+H\left(x_{\varepsilon}^{\delta} / \varepsilon, D \psi\left(x_{\varepsilon}^{\delta}, t_{\varepsilon}^{\delta}\right)+p_{\varepsilon}^{\delta}\right) \leq 0 \text { for any } p_{\varepsilon}^{\delta} \in \partial w_{\delta}\left(x_{\varepsilon}^{\delta}\right) .
$$

Since $w_{\delta}$ is inf-convolution of $w$, and $w$ is supersolution to the equation (20), we further have

$$
H\left(y^{\delta} / \varepsilon, D w_{\delta}(x / \varepsilon)+D \psi\left(x_{0}, t_{0}\right)\right) \geq \bar{H}\left(D \psi\left(x_{0}, t_{0}\right)\right)
$$

at any $x$ where $w_{\delta}$ is differentiable, with $y^{\delta}$ denoting the $w_{\delta}$-optimal point for $x$. Consequently

$$
H\left(y_{\varepsilon}^{\delta} / \varepsilon, q_{\varepsilon}^{\delta}+D \psi\left(x_{0}, t_{0}\right)\right) \geq \bar{H}\left(D \psi\left(x_{0}, t_{0}\right)\right)
$$


for any $\varepsilon$, some $q_{\varepsilon}^{\delta} \in \partial w_{\delta}\left(x_{\varepsilon}^{\delta}\right)$, with $y_{\varepsilon}^{\delta}$ denoting an $u_{\delta}$-optimal point for $x_{\varepsilon}^{\delta}$. In order to combine (23) and (24), we need to estimate

$$
\left|H\left(x_{\varepsilon}^{\delta} / \varepsilon, D \psi\left(x_{\varepsilon}^{\delta}, t_{\varepsilon}\right)+q_{\varepsilon}^{\delta}\right)-H\left(y_{\varepsilon}^{\delta} / \varepsilon, D \psi\left(x_{0}, t_{0}\right)+q_{\varepsilon}^{\delta}\right)\right|=: I .
$$

After straightforward computations, we get

$$
\begin{aligned}
I & \leq\left|D \psi\left(x_{0}, t_{0}\right)-D \psi\left(x_{\varepsilon}^{\delta}, t_{\varepsilon}^{\delta}\right)\right|\left(1+\left|V\left(x_{\varepsilon}^{\delta} / \varepsilon\right)\right|\right) \\
& +\left|D \psi\left(x_{0}, t_{0}\right)+q_{\varepsilon}^{\delta}\right| L_{V} \frac{\left|x_{\varepsilon}^{\delta}-y_{\varepsilon}^{\delta}\right|}{\varepsilon} \\
& \leq \mathrm{O}(\varepsilon)+\frac{\mathrm{o}(\sqrt{\delta})}{\varepsilon}+\frac{\mathrm{o}(\delta)}{\delta} \frac{1}{\varepsilon} .
\end{aligned}
$$

We freeze $\varepsilon$ and move $\delta$ in order to obtain a $\delta_{\varepsilon}$ for which

$$
I \leq \mathrm{O}(\varepsilon)+\frac{\varepsilon^{2}}{\varepsilon}+\frac{\varepsilon^{2}}{\varepsilon}=\mathrm{O}(\varepsilon) .
$$

We use the above estimate plus (23), (24) to finally get

$$
\begin{aligned}
0 & \geq \psi_{t}\left(x_{\varepsilon}^{\delta_{\varepsilon}} /, t_{\varepsilon}^{\delta_{\varepsilon}}\right)+H\left(x_{\varepsilon}^{\delta_{\varepsilon}} / \varepsilon, D \psi\left(x_{\varepsilon}^{\delta_{\varepsilon}}, t_{\varepsilon}^{\delta_{\varepsilon}}\right)+q_{\varepsilon}^{\delta_{\varepsilon}}\right) \\
& \geq \psi_{t}\left(x_{\varepsilon}^{\delta_{\varepsilon}}, t_{\varepsilon}^{\delta_{\varepsilon}}\right)+H\left(y_{\varepsilon}^{\delta_{\varepsilon}} / \varepsilon, D \psi\left(x_{0}, t_{0}\right)+q_{\varepsilon}^{\delta_{\varepsilon}}\right)-\mathrm{O}(\varepsilon) \\
& \geq \psi_{t}\left(x_{\varepsilon}^{\delta_{\varepsilon}}, t_{\varepsilon}^{\delta_{\varepsilon}}\right)+\bar{H}\left(D \psi\left(x_{0}, t_{0}\right)\right)-\mathrm{O}(\varepsilon) .
\end{aligned}
$$

Sending $\varepsilon \rightarrow 0$, we see that $u$ satisfies the subsolution test at $\left(x_{0}, t_{0}\right)$ with respect to the supertangent $\psi$, as was claimed.

Finally, taking into account that $(\overline{\mathrm{HJ}})$ satisfies a comparison principle, we get that the $u_{\varepsilon}$ locally uniformly converge to $v=u$.

\section{References}

1. Alvarez, O., Bardi, M.: Ergodicity, stabilization, and singular perturbations for Bellman-Isaacs equations. Mem. Am. Math. Soc. 204, 1-88 (2010)

2. Armstrong, S.N., Souganidis, T.: Stochastic homogenization of level-set convex Hamilton-Jacobi equations. Int. Math. Res. Not. IMRN, 3420-3449 (2013)

3. Cardaliaguet, P., Nolen, J., Souganidis, P.E.: Homogenization and enhancement for the G-equation. Arch. Rat. Mech. Anal. 199(2), 527-561 (2011)

4. Evans, L.C.: The perturbed test function method for viscosity solutions of nonlinear PDE. Proc. R. Soc. Edinb. Sect. A 111(3-4), 359-375 (1989)

5. Davini, A., Siconolfi, A.: Weak KAM theory topics in the stationary ergodic setting. Calc. Var. Partial Differ. Equ. 44, 319-350 (2012)

6. Fathi, A., Siconolfi, A.: PDE aspects of Aubry-Mather theory for quasiconvex Hamiltonians. Calc. Var. Partial Differ. Equ. 22(2), 185-228 (2005)

7. Lions, P.-L., Papanicolaou, G., Varadhan, S.R.S.: Homogenization of Hamilton-Jacobi Equations. unpublished manuscript, circa (1986)

8. Ros, A.: The isoperimetric problem. In: Proceedings of the Clay Mathematical Institute-MSRI summer school on Minimal Surfaces (2005)

9. Xin, J., Yu, Y.: Periodic homogenization of inviscid G-equation for incompressible flows. Commun. Math. Sci. 8(4), 1067-1078 (2010) 